\title{
An Evolutionary Disc Model of NGC 5907
}

\author{
Andreas Just ${ }^{1}$, C. Möllenhoff ${ }^{2}$, and A. Borch ${ }^{1}$ \\ ${ }^{1}$ Astronomisches Rechen-Institut am ZAH, Mönchhofstraße 12-14, 69120 Heidelberg, Germany \\ email: just@ari.uni-heidelberg.de \\ ${ }^{2}$ Landessternwarte am ZAH, Königsstuhl 12, 69117 Heidelberg, Germany \\ email: C.Moellenhoff@lsw.uni-heidelberg.de
}

\begin{abstract}
We present the first physical disc model of NGC 5907, which reproduces simultaneouly the multi-band photometry in U, B, V, R, and I- band (Just et al., 2006, A\&A in press, astro-ph/0608196). The model is based on the star formation history and dynamical evolution of the disc and includes dust extinction consistent with the dust properties derived from FIR observations. We also find naturally the stellar light eccess of the young sub-population near the midplane (hidden by the dust), which is required to heat the dust.
\end{abstract}

Keywords. stellar dynamics, galaxies: evolution, stellar content, structure, ISM: dust extinction

The stellar disc model is constructed by a sequence of sub-populations according to the SFR and the heating function, which are in dynamical equilibrium with the gravitational forces of stars, gas and dark matter halo. The intrinsic luminosity is derived by the stellar population synthesis code PEGASE.

We performed deep photometry in U, B, V, R, I bands using the $2.2 \mathrm{~m}$ telescope of the Calar Alto Observatory, Spain. With the CAFOS focal reducer the field-of-use had a diameter of 16 arcmin. From subsequent vertical stripes parallel to the minor axis we derived surface brightness and colour profiles, which are simultaneously fitted by the model profiles. The final model has a moderate decline in the star formation history
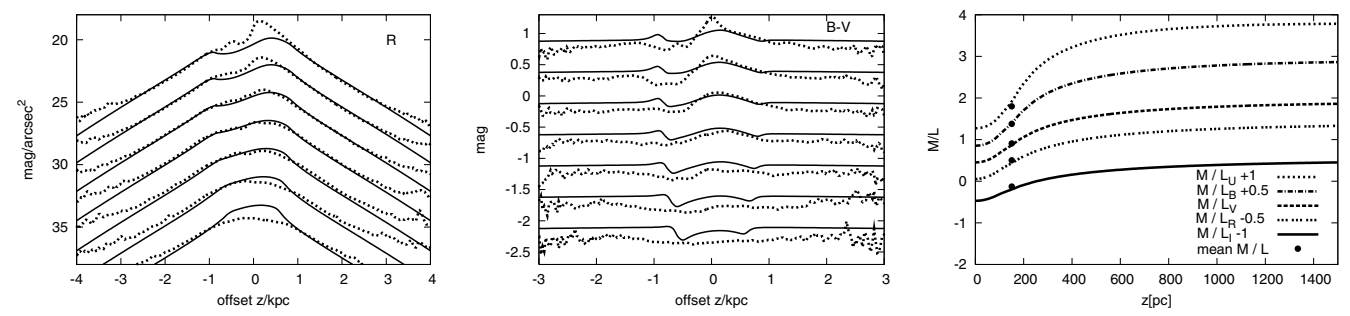

Figure 1. Comparison of observed (dashed) and model (full) surface brightness and colour profiles. Vertical cuts start at the minor axes and are shifted parallel by 45 arcsec along the major axes (top to bottom). R-band profiles (left) are shifted by $2 \mathrm{mag} / \operatorname{arcsec}^{2}$ and colour profiles (middle) by $1 \mathrm{mag}$ from one to the next. Intrinsic mass-to-light ratios (right, in solar units) show significant variations with height above the midplane.

with time and a linear increase of the velocity dispersion with age of the stellar subpopulations. The stellar disc has slightly larger intrinsic scale length and scale height as in earlier investigations, which is mainly an effect of the cutoff. We used a Scalo IMF resulting in a relatively low stellar disc mass with a mass-to-light ratio of 0.91 in $\mathrm{V}$ band. The total mass of the dust component is only $5 \%$ smaller than that required from FIR/submm observations. We find a significantly larger scale length for the dust, which fits well to the HI distribution. 